V. Dignum, Towards a People-oriented Knowledge Management Environment, Proceedings of DEXA2000, A.M. Tjoa, R.R. Wagner,A.Al-Zobaidie(eds), 11th International Workshop on Database and Expert Systems Applications, IEEE Computer Society, London, September 2000. 


\title{
Towards a People-Oriented Knowledge Management Environment
}

\author{
Virginia Dignum \\ NOTION - University Nyenrode/Achmea \\ v.dignum@nyenrode.nl
}

\begin{abstract}
In this preliminary report we present ongoing research on intelligent knowledge management (KM) environments supporting communication in a virtual environment. An agent community handles the interaction between knowledge sources of different degrees of formality and knowledge users and creators, based on real user needs and virtual collaboration. Intelligent agents handle knowledge sources and tasks, and a personal assistant provides a personalised, dynamic interface to users. The concept of IKME is being applied in the context of a cross sell support pilot at Achmea Customer Care Centre in the Netherlands.
\end{abstract}

\section{Introduction}

This paper describes ongoing research on a dynamic, intelligent knowledge management environment (IKME), in which an agent community assists in collaboration and interaction activities between knowledge sources, applications and users. We introduce a methodology to support organisational learning in a virtual environment, based on task-oriented, personalised interface providing uniform access to a diversity of knowledge and information sources of different degrees of formality.

In section 1, we define our view of $\mathrm{KM}$ and intelligent systems in virtual business processes. In section 2 we introduce our approach to modelling an intelligent $\mathrm{KM}$ environment, taking human computer interaction issues into account. Section 3 presents the concept of an intelligent KM environment. Initial ideas on the role of agents in this environment are presented in section 4. Section 5 briefly describes a project being developed at Achmea using the IKME framework. Finally, we present conclusions and discuss future work.

\section{KM systems for virtual organisations}

In order to accommodate the fast pace of technological change, global competition and the emergence of a knowledge-based economy, many organisations are in the midst of fundamental changes in organisational design and management practices. The result must be a flexible and agile organisation where innovation, creativity and change are possible. Flexibility is a multidimensional concept - demanding agility and versatility; associated with change, innovation and creativity; coupled with robustness and resilience, implying stability, sustainable advantage and capability to evolve over time [2].

People are the key actors in the organisational processes and the main users of information systems. Complex demands of modern organisations make it imperative that workers share different types of knowledge in their work. Knowledge is therefore a modern organisation's most important asset. KM activities include the identification, acquisition, preservation, dissemination and use of enterprise knowledge to be able to respond to rapid changes in a knowledge-based economy. So far, many KM efforts have been technology-driven. Technical advances open up large, heterogeneous amounts of information, creating an 'everybody wants to know everything' syndrome. Humancentred, intelligent KM systems are needed, that can assist workers in generating 'just in time' and 'just enough' knowledge, prevent information overload and keep workers stimulated to share relevant knowledge in a dynamic, collaborative environment.

Only a decade ago, information systems were build to serve a specific purpose, within a single department or group. In order to support modern organisations effectively, information systems now must be able to access and combine information from different sources. Information systems to support modern business applications must be decentralised, autonomous and heterogeneous. A new generation of information systems is shifting towards the integrated support of structured and unstructured processes and information sources, formal and informal communication and different levels of activity coordination [3]. Moreover, such support should be dynamic, personalised and adaptive to change.

\subsection{From Information Systems to KM Systems}

Over the years, computer science disciplines have started to develop methodologies and applications to support intelligent information storage as well as task specific support systems. Information systems help people analyse 
problems, visualise complex subjects, and create new products. Lately, the role of information systems has shifted from the support of one specific function and set of users, to that of supporting collaboration and business processes in a decentralised, distributed environment [10]. Moreover, support is becoming more dynamic, personalised and adaptive to change.

The growing importance of distribution of knowledge and information has given rise to the development of several software systems for the support of distributed KM within an organisation such as Document Management Systems (DMS), GroupWare, and Intranets and Extranets. These systems have improved information availability, but have a number of weaknesses [4]. Existing systems mainly concentrate on explicit knowledge, leaving tacit knowledge outside the system, so that integral experience based knowledge is not shared. Knowledge is often considered out of context, limiting its usefulness to people with background (implicit) knowledge. People need to have already contextual knowledge in order to use the information system effectively. Furthermore, in order to extract added value from stored information, additional tasks have to be performed that have no immediate value. Often the meaning of terms is not explicitly stored in the system. As meanings of words change over time, stored knowledge is misunderstood. Most systems do not provide generic solutions or support knowledge combination across organisational boundaries and often do not make use of interactive media and virtual business processes.

Organisational knowledge is usually embedded in information systems and not handled formally by the system. In the last years considerable effort has been made, by different computer science disciplines to develop methodologies and applications to support both in the area of intelligent information gathering and storage as in the area of task specific support systems.

Organisational memories are motivated by the desire to preserve and share the knowledge and experiences that reside in an organisation. An organisational memory can be seen as a shared information system: a space of meanings, terminologies, practices, understandings, cultural norms, shared values in an essentially human oriented network within which artificial agents and technologies play an important support role [5]. In this way, an organisational memory, integrating people and technology, can be seen as a cognitive system, that is 'a complex information processing system that perceives, solves problems, learns, and communicates.' [11]. These systems actively supports users working on knowledge intensive tasks by providing necessary and useful information for the task.

\section{Knowledge management environment}

Although traditional information systems provide support to knowledge workers in their daily work, a environment is needed that integrates the business process aspects of knowledge work with active support for using and adding to heterogeneous knowledge sources [9]. KM environments must be proactive, that is, taking initiatives in a goal-oriented way as well as reactive, that is, respond to user requests or environment changes. The main goal of a $\mathrm{KM}$ environment is to provide relevant knowledge to assist the human user in executing knowledge intensive tasks. To be effective, such environments must provide users with relevant knowledge at the right time. By relevant knowledge we mean knowledge which enables users to perform their tasks better with this knowledge than without it. To be accepted by users, the environment must be able to adapt to the different needs and preferences of users, and integrate naturally with existing work methods, tools and processes. The KM environment relies on an explicit modelling of business processes, such as conventional business process models and workflow management systems.

Heterogeneous knowledge environments are open and might change rapidly over time. Because knowledge is embedded in a multitude of sources, KM systems should be able to handle formal and informal knowledge representations, as well as heterogeneous multimedia knowledge sources. The knowledge assets available in a KM environment are more than 'traditional' information systems alone. Such assets include structured and unstructured information, multimedia knowledge representations and links to people (ex. through knowledge maps or yellow pages). Besides using existing knowledge sources, the environment should be able to create (and store) new knowledge based on its observation of the user's task performance [7].

Frameworks such as CORBA and DCOM were developed to support distributed computing in heterogeneous environments. Such frameworks encapsulate the heterogeneity of legacy systems and applications within standard, interoperable wrappers. These frameworks are well suitable to the 'data' level of communication.

The problem faced at the knowledge level is to develop a higher level of integration based on the semantics of the problem at hand. It is necessary to create a dynamic relationship between knowledge-intensive business processes and knowledge sources. In order to be able to support the execution of knowledge-intensive tasks, using knowledge from heterogeneous sources, according to diverse user preferences, a common knowledge description must be available, as well as a means to 'translate' domain concepts and relationships between heterogeneous participants.

Furthermore it is necessary to separate the use of knowledge from the specific characteristics of the knowledge source. Integration is achieved in a knowledge sharing environment by providing uniform access to a knowledge and information sources of different degree of 
formality. This environment should include loosely connected heterogeneous, multimedia sources, dynamically defined goals, virtual, dynamic links between knowledge needs and knowledge sources, and, adaptable, intelligent personal assistants, providing support to users.

\section{Agent based KM}

We propose a functional separation between knowledge use and knowledge sources as a way to incorporate dynamic behaviour into information systems design. Due to their characteristics intelligent software agents are strong candidates for acting as mediators between source and application of knowledge. A software agent is an autonomous computational entity with communication capabilities (social), able to react to environmental changes (reactive) and act in a goaloriented way (proactive) [12]. Agents can check of the dynamic conditions of the environment, reason to interpret those perceptions, solve problems, draw inferences and determine actions, and finally, act accordingly.

\subsection{Knowledge sharing}

One of the main aims of $\mathrm{KM}$ is to provide an environment for optimal sharing of knowledge between agents. In the context of our research agent can refer to both human and software agents. Knowledge sharing is basically done in two ways [8]:

- Socialisation: Sharing of tacit knowledge between agents. In this way knowledge moves from tacit to tacit.

- Articulation: An individual formulates his own tacit knowledge in a way that can be communicated to others.

Most KM efforts focus on the articulation, or formalisation, of knowledge, that is in the conversion of tacit, personal knowledge into explicit, organisational knowledge. As anyone who has been involved in the development of expert systems and knowledge based systems can tell, the cost of formalising knowledge is very high and the resulting solution is not always very useful (rate at which knowledge becomes obsolete or useless). Parts of corporate knowledge that needs to be processed by computer must be formalised, but other parts that are mainly to be understood by human users can be left informal [1]. However, a common knowledge description formalism is needed such that different agents can share knowledge indicators, such as an ontology. Ontologies aim at capturing domain knowledge in a generic way and provide a commonly agreed understanding, which may be reused and shared across applications and groups. Ontologies provide a common vocabulary of an area and define - with different levels of formality - the meaning of the terms and the relations between them [6].
In our view, knowledge sharing efforts should concentrate on the describing the context of knowledge rather than in the knowledge contents. We envision an environment in which knowledge use starts from the current knowledge needs in a given business process. Different knowledge intensive tasks within a process will use knowledge from different sources. In order to share knowledge across tasks and processes, it is necessary to use some sort of indicator to point out applicable knowledge independently from the type of knowledge (formal and informal, tacit, explicit, etc.).

This environment provides a uniform view of heterogeneous knowledge sources by defining a level of knowledge description in which knowledge contents are not necessarily always formalised. Knowledge sharing is done at the level of knowledge description. Only when an agent decides to use a certain 'piece' of knowledge, it will then look for the actual knowledge content. Instead of 'offering' knowledge contents by formalisation, in this system knowledge contents are accessed on request.

\subsection{IKME architecture}

We propose a dynamic, intelligent knowledge management environment (IKME), in which knowledge can be shared and used in a way that is transparent to all workers and easily accessible to the organisation as a whole. The environment is more than a passive information system and actively provides intelligent assistance to the user.

In our view, the emphasis of any KM environment must be the support of the (collaboration between) human users on their knowledge-intensive tasks by providing, maintaining and distributing relevant knowledge. The following requirements are essential to the practical success of such an environment [1]:

- Collection and organisation of knowledge from distributed, heterogeneous sources. This does not mean a central knowledge repository, but, the development of a system of knowledge 'indicators', based on the description of the knowledge sources and not in the formalisation of their contents.

- Minimisation of up-front knowledge engineering. Users have little time to spare for formal requirements and knowledge acquisition. Therefore, the environment must exploit existing knowledge, provide quick benefits and adapt to changing requirements.

- Exploit user feedback for learning and evolution. Maintenance efforts by the user should be minimised. The environment must be able to use user's feedback and reaction to learn and improve its own knowledge.

- Integration to existing work environments. The environment must tap from existing organisation flows of knowledge instead of creating a complete new flow. At the technical level this means that the system must be able to interface directly to existing tools. 
- Active presentation of relevant knowledge. The system should actively use its (growing) knowledge about the user interests, goals and learning style to provide useful information and be a competent partner for cooperative problem solving.

- Active preservation of relevant results. Knowledge capture and creation must be fully integrated into the support of user's tasks. The system must be capable of identifying and recording new knowledge created by the user during its interaction with the system.

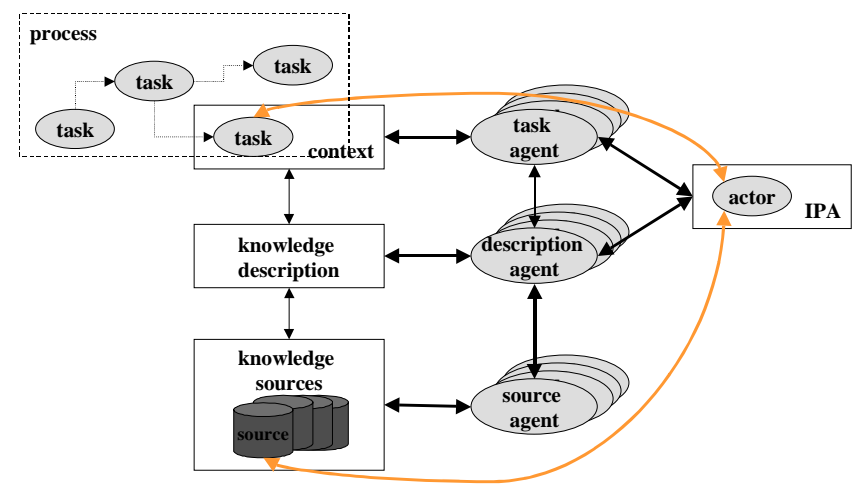

Figure 1 - IKME architecture

An important consequence is that any architecture implementing these requirements cannot be completely formally specified before hand. In this architecture the specification of application, description and representation of knowledge are separated. A community of collaborative agents will be responsible for the (goaloriented) interrelation of the different layers. However, a framework for collaboration is needed describing the knowledge context, that is, as a common 'belief space' of all agents. By collaborating with each other and with users, the agents will learn and dynamically extend this framework. In order to assure the feasibility of the KM environment a balance must be found between collaboration and agent freedom. That is, a belief-space that is too small will increase agents' freedom but limit learning, because there will be not enough ground for meaningful collaboration. Contrariwise, a belief-space that is too large will be too inflexible to organisational changes and limit the agent's freedom of action.

Consequently, we propose an architecture with two parts: (1) a basic framework in which knowledge sources, context and applications are described, and (2) a community of intelligent agents, which will construct a dynamic architecture by means of collaboration. Fig. 1 gives an overview of the proposed architecture.

The intelligent personal assistant (IPA) reflects the personal preferences, learning style and work process of the knowledge worker. The IPA functionality should ideally include giving advice, provide intelligent assistance and tutoring, support activity planning and collaborative work. The purpose of knowledge access/creation is described at the task description level. At this level, problems to be solved and tasks to be executed are represented and planned. Task level agents have two main roles: the identification of knowledge needs at task and process level, and the identification of task patterns and behaviours for possible reuse while also communicating with other task and IPA agents to share experiences and knowledge. The knowledge description level must enable a dynamic, uniform and intelligent, access to the different knowledge sources. The knowledge description level gives a uniform description of the context of the knowledge sources. The description framework used should be able to dynamically adapt to changes in the environment (sources, goals, tasks and user preferences). In order to plan and realise tasks in the task description level the IPA will use the community of agents at the knowledge navigation level, to identify and use the objects in the knowledge source level, based on their description represented in the knowledge description level. This happens in collaboration with the user and taking user's wishes and preferences into account. Finally, the knowledge source level contains heterogeneous, autonomous, decentralised, multimedia and multi-modal sources. Examples are structured and unstructured documents, databases, recordings of conferences, links to people, etc. Source agents are responsible for the access/update of the different sources.

We assume that agents involved are collaborative and social, that is, their actions are meant to maximise the benefit to the community and will share their knowledge.

The user should still be free to access knowledge sources and perform tasks directly. In this case, the IPA should 'look over the user's shoulder', recording user actions for possible sharing and reuse. For example, if Mary used her assistant to support her gathering knowledge about representing client profiles, the IPA should afterwards be able to a) identify Mary as someone who probably knows about client profiles, and, b) share the process Mary followed and her conclusions with other seekers of 'similar' knowledge. This functionality can be achieved when the IPA is embedded in the interface of existing tools.

\section{An IKME to support cross sell activities}

A system to support cross sell activities in the Customer Care Centre of a daughter company of Achmea (an insurance and banking firm) is being developed as 'proof of concept' for the IKME framework. Achmea call centres handle several million calls per year, dealing with marketing and sales, mutations of client portfolios and damage reports. Currently call centres are specialised in different products. Lately, a lot of the client contact has shifted from telephone to Internet. Integration of all client contact systems is the aim of the global Customer Care Centre. Furthermore, Achmea aims to extend their cross sell activities, by providing adequate support and identification of opportunities. Knowledge in a call centre is embedded in a 
multitude of different sources, including people, telephone call handling systems, flexible scripting systems, databases, e-mail and internet. An ideal IKME for a call centre must be able to handle structured or unstructured information, multimedia knowledge representations and links to people (filtering calls, email, knowledge maps or yellow pages). To be accepted by human call centre agents, the system must adapt to the needs and preferences of users, and integrate naturally into existing work methods, tools, and interfaces.

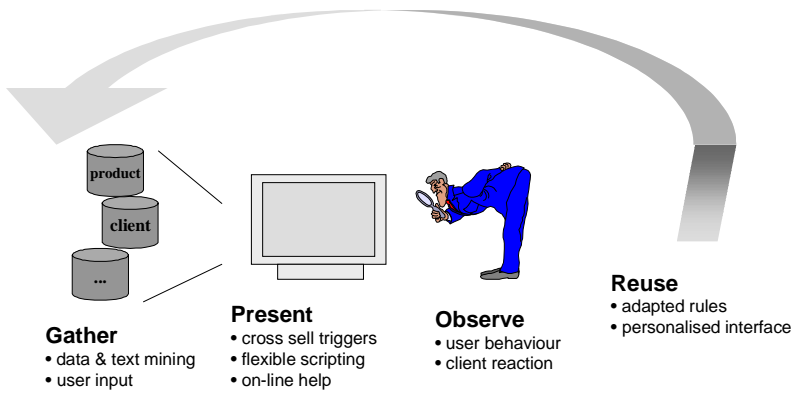

Figure 2 - Customer Care Assistant

Furthermore, the cross sell process must adapt to the client needs and background. Exiting cross-sell rules, based on product specifications, should then be dynamically refined based on client preferences and observations of call centre employees. In this way, the system will learn from the application of the increasingly refined rules. Fig. 2 illustrates the phases of the process.

\section{Conclusions and future work}

We have introduced our view on the role of agents in the design and functionality of information systems as $\mathrm{KM}$ environments. The IKME system presented is able to provide uniform access to a diversity of knowledge and information sources of different degree of formality using agents for communication between knowledge sources and consumers. We believe that the main consequences of our modelling approach for future research in KM and collaborative systems are as follows:

1. It is fundamental to consider human-computer interaction principles in order to produce a successful human-centred design of a KM environment.

2. KM systems cannot be implemented based on completely formal specifications, but must seek a balance between the description of knowledge sources and evolving business applications.

3. A methodology is needed for the development of a framework for collaborative learning in a virtual environment, based on intelligent agents.

4. A balance between collaboration and agent freedom will assure the feasibility of the KM environment.
In the course of our project, several prototypes will be implemented in different Achmea business units. Areas being considered for the implementation of prototypes include an online personal insurance manager, support of the insurance acceptation process, etc.

\section{References}

[1] Abecker, A., Bernardi, A., Hinkelmann, K., Kühn, O. and Sintek, M., "Towards a Technology for Organizational Memories", IEEE Intelligent Systems \& Their Applications, 13(3). 1998.

[2] Bahrami, H., "The emerging Flexible Organisation: Perspectives from Silicon Valley", reprinted in Myers, P. (ed.) 1996, Knowledge Management and Organisational Design, Resources for the Knowledge-based Economy, ButterworthHeinemann, 1992.

[3] Dignum, F. \& Houben, G.J., "Integrating Information Systems: linking global business goals to local database applications", Trans. Society for Design and Process Science, 3(1), 1999, pp. 4353.

[4] Dignum, V. and Heimannsfeld, K., "Knowledge Management for Requirements Engineering", Proc. of KAW99, Banff, Canada, http://sern.ucalgary.ca/KSI/KAW/KAW99/papers.html, 1999.

[5] Gammack, J., "Organisational Memory and Intelligent Decision Support in Shared Information Systems", Research Working Paper IT/98/05, Department of Information Technology, Murdoch University, http://www.it.murdoch.edu.au, 1998.

[6] Gomez Perez, A. and Benjamins, V.R, "Overview of Knowledge Sharing and Reuse Components: Ontologies and Problem-Solving Methods", Proc. of KRR5, IJCAI-99, V.R. Benjamins, B. et. al. (eds.), Stockholm, 1999.

[7] Leake, D., Birnbaum, L., Hammond, K., Marlow, C. and Yang, H., "Task-Based Knowledge Management", Proc. of the AAAI Workshop on Knowledge Management and Case-Based Reasoning, Orlando, AAAI Technical Report WS-99-10, 1999.

[8] Nonaka, I., "The Knowledge-Creating Company", reprinted in 1998 in Harvard Business Review on Knowledge Management, Harvard Business School Press, 1991.

[9] Staab, S. and Schnurr H-P., "Knowledge and Business Processes: Approaching an Integration", Proc. of OM'99, IJCAI99, Stockholm, 1999.

[10] Verharen, E., A Language-Action Perspective on the Design of Cooperative Information Agents, $\mathrm{PhD}$. thesis, University of Tilburg, The Netherlands, 1997.

[11] Webster, C., "Communication Ethics and HumanComputer Cognitive Systems", Proc. $1^{\text {st }}$ Int. Conf. on Cognitive Technology, 1995.

[12] Wooldridge, M. and Jennings, N., "Intelligent agents: Theory and Practice", The Knowledge Engineering Review, 10(2), 1995, pp.115-152. 